\title{
NUMERICAL CALCULATION OF THE ELECTROSTATIC PO'TENTIAL AROUND AN IONIC MICELLE WITH COUNTER ION BINDING
}

\author{
Sze-Tsen LeE (李世琛) AND CHUng YUAN MOU* (倿中原) \\ Department of Chemistry, National Taiwan University, \\ Taipei, Taiwan 107, R.O.C.
}

Key Word Index-Poisson-Boltzmann equation; micelle; counterion binding.

\begin{abstract}
A numerical solution of the nonlinear Poisson-Boltzmann equation (PBE) is presented for a system of spherical micelles with counterion binding. The approach investigates the following effects on ion-micelle interactions, (i) total surface charge, (ii) competitions of different counter ions on micellar surfaces and (iii) surface potential determination. The theory is applied to interpret the ion activities in micellat solution as measured by ion-sclective electrodes.
\end{abstract}

In recent years, physical chemists and biochemists have become more interested in the interactions and reactions that take place at charged surfaces in solution, for instance, on ionic micelles, microemulsions, vesicles and membranes.

In these systems, it is very important to understand the interaction of the micellar surface and its counterions, that is, the distribution of ions around the charged surface. This is necessary to understand phenomena such as micelle catalyzed chemical reactions'), the stability of colloids ${ }^{2}$ and the free energy of charged surface systems".

Among the experimental techniques used in investigating counterion-charged surface interactions, the simplest one may be the measurement of the thermodynamic activities of the counterion by the ion-selective electrode method"). In this technique, one measures the decrease of ion activity due to its strong adsorption on the charged surface. Usually, the electrode responds only to the activity of a selected ion following the Nerst law over several decades of ion concentration.

For micellar solutions, the problem can often be simplified to point-like charges interacting with a spherical macroion. The adsorption can be divided into surface (Stern layer) adsorption and electric double layer adsorption ${ }^{5}$. Therefore, a detailed knowledge of the ion distribution in the vicinity of charged surfaces is necessary for a complete understanding of the processes involved.

In this paper, our interest is on a mixed-electrolyte system, an ionic micelle and a simple electrolyte. There are at least two kinds of counterion exchanges on the micellar surface. The surface potential is determined by both kinds of ions.

In principle; the measured ion activity of a certain ion reflects its adsorption both on the Stern layer and on the electric double layer. For the double layer, the relation between the electric field $\vec{E}$ and the distribution of the charge $\rho$ is described by the Poisson equation

$$
\vec{\nabla} \cdot\left(D \epsilon_{0} \vec{E}\right)=\rho
$$

where $\vec{E}$ is given by

$$
\vec{E}=-\vec{\nabla} \phi
$$

* Present address: Defartment of Chemistry, National Tsing-Hwa University, Hsinchu, Taiwan 300, R.O.C. 
$D$ is the dielectric constant, $\epsilon_{0}$ is the vacumn permittivity, and $\phi$ is the electric potential.

With a Boltzman distribution of charges, we have the Poisson-Boltzmann equation

$\nabla^{2} \phi(r)=-\frac{1}{D \epsilon_{0}} \Sigma_{\alpha} e Z_{\omega} C_{\alpha} \exp \left[-\frac{e Z_{\alpha} \phi(r)}{k_{B} T}\right]$

where $k_{B}$ is the Boltzmann constant and $C_{\alpha}$ is the concentration of the $\alpha$ th ion.

Evidently, there are no exact analytical solutions for Poisson-Boltzmann equation for spherical system. The purpose of this paper is to devise a numerical procedure for the solution of the Poisson-Boltzmann equation. The approach will answer the following questions: (i) What is the effect of the total surface charge on the adsorption behavior? (ii) What is the effect of the competition between different counterions on the micellar surface? (iii) How is surface potential to be determined? With these aims, we emphasize the coulombic interaction and the chemical exchange on the surface. The short-range potentials ${ }^{6)}$, hydration forces" and the compressibility of the solvent ${ }^{\text {) }}$, are neglected.

Previously, Bentz" had considered an approximate closed-form solution to PBE with cation binding, their method, however, is limitted to larger spheres ( $\operatorname{radii}<10 \kappa^{-1}$ ). Frahm and Diekmann ${ }^{10}$ ) also considered a numerical approach to a modified PBE which is suitable to micellar systems. Our approach is similar to that of Frahm and Diehmann, however we wish to study more explicitly the influence on the surface potential of mixed electrolytes in competition in the Stern layer. In this initial study, we have neglected the field dependence of the dielectric constant. This will be the subject of later work.

\section{THEORY}

We will consider our micelle as a sphere of radius $a$ consisting of $g$ surfactant molecules. The solution consists of simple ions with concentrations $C_{\alpha}$. Thus the Debye screening constant $\kappa$ is

$$
\kappa^{2}=\frac{e^{2}}{D \epsilon_{0} k_{B} T} \Sigma_{\omega} Z_{\omega}^{2} C_{\infty}
$$

where $Z_{a}$ is the charge of the $\alpha$ th species.

In terms of the reduced variables

$$
\begin{aligned}
& \mu=\kappa R-\kappa a \\
& \emptyset(\mu)=e \phi / k_{B} T,
\end{aligned}
$$

the Poisson-Boltzmann equation can be written as

$$
\begin{aligned}
& \frac{d^{2} \Phi}{d \mu^{2}}+\frac{2}{(\mu+\kappa a)} \frac{d \Phi}{d \mu} \\
& =-\frac{e^{2}}{\kappa^{2} D \epsilon_{0} k_{B} T} \sum_{\infty} Z_{\alpha} C_{\alpha} e^{-Z_{\omega} \Phi} \\
& =-\frac{1}{2 I} \Sigma_{\alpha} Z_{\alpha} C_{\alpha} e^{-Z_{\omega}}
\end{aligned}
$$

with $I$ as the ionic strength

$$
I=\frac{1}{2} \Sigma_{a} Z_{a}^{2} C_{a} \text {. }
$$

The boundary condition of the PBE will be determined by the surface charge:

$$
\begin{aligned}
& \left.\left(\frac{d \phi}{d r}\right)_{r=a}=-\frac{\sigma}{D \epsilon_{0}}, \quad \phi(r=a)=\phi_{0}\right) \\
& \text { or } \\
& \left.\left(\frac{d \Phi}{d \mu}\right)_{\mu=0}=-\frac{\sigma e}{\kappa k_{B} T D \epsilon_{0}}, \emptyset(\mu=0)=\emptyset_{0}\right)
\end{aligned}
$$

where $\sigma$ is the surface charge density

$$
\sigma=\frac{q}{4 \pi a^{2}}
$$

determined by the degree of counterion binding. The assymptotic value of $\phi(r)$ is of course zero,

$$
\lim _{r \rightarrow \infty} \phi(r)=0 \text {. }
$$

Let us consider a solution of surfactant $M_{a} P$ with concentration $C_{a}$ and an added strong electrolyte $M_{b} Q$ with concentration $C_{b}$. $\quad M_{a} P$ forms micelles of $g$ aggregates at critical micelle concentration (CMC), $C_{M}$. Let the degree of dissociation of $M_{\text {。 }}$ 
at the micellar surface be $\alpha$, and $\beta$ be the number of $M_{b}$ ions adsorbed per micelle, including both the Stern layer and the double layer. Then one has

$$
\begin{aligned}
& {\left[M_{s}\right]=C_{M}+\alpha\left(C_{s}-C_{M}\right)} \\
& {[P]=C_{M}}
\end{aligned}
$$

assuming that the monomer concetration of $M_{a} P$ is constant beyond the CMC. One also has

$$
\begin{aligned}
& \left.\begin{array}{l}
{\left[M_{b}\right]_{0}=C_{b}} \\
{[Q]=C_{b}}
\end{array}\right\} \\
& \text { [micelle }]=\left(C_{s}-C_{M}\right) / g
\end{aligned}
$$

where $\left[M_{b}\right]_{0}$ represents the "unperturbed" concention of $M_{b}$ if no adsorption of ions on micelle occurs.

Now $\beta$ is related to the experimentally measured concentration of $M_{b},\left[M_{b}\right]$, through an electrode responding to $M_{b}$ only by

$$
\beta=\frac{\left[M_{b}\right]_{0}-\left[M_{b}\right]}{[\text { micelle }]}=\frac{\left\{C_{b}-\left[M_{b}\right]\right\} g}{C_{a}-C_{M}} .
$$

From Eqs. (10), (11), (12), $\alpha$ and $\beta$ can, in principle, be calculated after the ion concentrations (activities) $\left[M_{a}\right]$ and $\left[M_{b}\right]$ are measured using the approapriate selective electrodes.

The next step, then, is to relate $\alpha$ and $\beta$ to the surface potential $\phi_{0}$. The problem is to relate the percentage of $\beta$ in the double layer and the Stern layer. Let the percentage of adsorbed $M_{b}$ in the Stern layer be $\alpha_{b}$, the percentage in double layer will then be $1-\alpha_{b}$.

We consider a simple exchange mechanism

$$
\begin{gathered}
M_{a} \text { (Stern layer) }+M_{b} \text { (soln) } \rightleftarrows \\
\left.M_{a} \text { (soln) }+M_{b} \text { (Stern layer }\right)
\end{gathered}
$$

That is, a one-to-one replacement is postulated. Then Eq. (10a) should be revised as

$$
\left[M_{a}\right]=C_{M}+\alpha\left(C_{a}-C_{M}\right)+\alpha_{b}\left\{C_{b}-\left[M_{b}\right]\right\} .
$$

The last term is the part driven out from the micelle surface. Also, the concentra- tion of free $M_{b}$ ion in solution $C_{b}^{\prime}$ should be

$$
\begin{aligned}
C_{b}^{\prime} & =C_{b}-\alpha_{b}\left\{C_{b}-\left[M_{b}\right]\right\} \\
& =C_{b}\left(1-\alpha_{b}\right)+\alpha_{b} M_{b} .
\end{aligned}
$$

The surface charge and surface charge density of micelle will be given by $Z_{m}$ and $\sigma$, respectivel,

$$
\begin{gathered}
Z_{m}=-\left(\alpha g-\alpha_{b} \beta\right) \\
\sigma=Z_{m} / 4 \pi a^{2} .
\end{gathered}
$$

With Eqs. (15), (16), (17), one can start to solve the Poisson-Boltzmann equation and calculate the surface potential. This potential should be related to $\alpha, \beta$ and $\alpha_{l}$. In the next section, we will give a short discussion of the numerical procedure.

\section{NUMERICAL PROCEDURE}

In our solution of the PBE, the surface potential appears, at first, to be an adjustable parameter. One guesses $\phi_{0}$ initially and then solves the initial value problem to "shoot" the boundary condition $\emptyset(\infty)=0$. If the estimated $\varphi_{0}$ is not right, then we again iterate from $r=a$ until $\Phi(r \rightarrow \infty)=0$. For an initial guess, we use the zeroth order approximation

$$
\Phi(0)=2 \sinh ^{-1}\left[-\frac{1}{2}\left(\frac{d \Phi}{d \mu}\right)_{\mu=0}\right]
$$

for a flat surface.

The numerical quadrature we used is the 4th Runge-Kutta-Nyström method preceding in equidistant steps. The solution is judged to be good if the potential decreases to $0.1 \%$ of its initial value without go-away behavior. The accuracy of the procedure has been checked in two examples. We tested the pocedure on the Debye-Hückel formula and on a linearized Poisson-Boltzmann equation. We also tested it against a known analytical solution of the PBE for a flat surface. The deviations of $\phi(r)$ are less than $0.01 \%$ and for $\phi^{\prime}(r)$ less than $1 \%$.

One important part of our procedure 
is to test that electric neutrality is maintained. The total charge surrounding the micelle should be equal and opposite in sign to the charge on the micelle. That is,

$$
\int_{\sigma}^{\infty} 4 \pi r^{2}\left\{\Sigma_{a} Z_{\omega} C_{a} e^{-\frac{e Z_{n} \phi(r)}{k_{B} T}}\right\} d r=-Z_{m}
$$

or

$$
\begin{aligned}
& \frac{4 \pi}{\kappa^{3}} \int_{0}^{\infty}(\mu+\kappa a)^{2} \Sigma_{a} Z_{a} C_{\infty} \exp \left(-Z_{a} \Phi(\mu)\right) d \mu \\
& \quad=-Z_{m} .
\end{aligned}
$$

The electric neutrality condition is generally satisfied within $0.6 \%$ error.

All the calculations in this paper are done on an Apple II microcomputer. Programs are available upon request.

\section{RESULTS AND DISCUSSIONS}

First, we consider a simple model system of pure micelle formed from $M_{a} P$ with no salt added. In this model calculation; the aggregation number is 70 , the degree of dissociation $\alpha=0.10$, and $a=15 \AA$. We calculated two cases of ionic strength, $0.01 M$ and $0.001 M$. A typical potential versus distance curve is shown in Fig. 1. The calculated surface potentials are

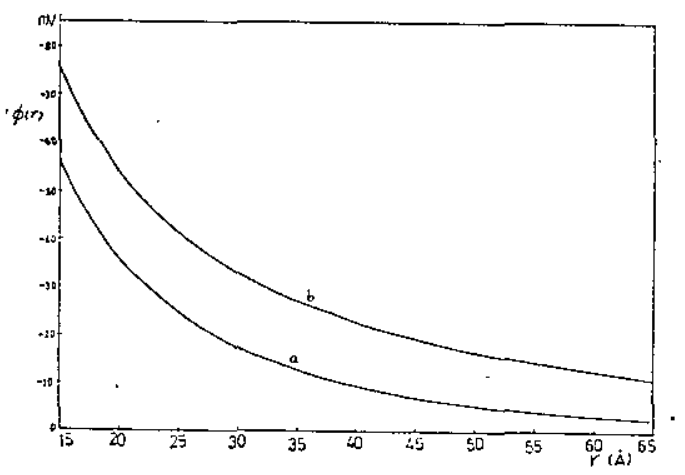

Fig. 1. Potential versus distance $a$ for model micelle system of simple $M_{\alpha} P$ with no $M_{6} Q$ added.

$g=70, \alpha=0.10, a=15 \AA$

Ionic strength: (a) $I=0.01 \mathrm{M}$

(b) $I=0.001 \mathrm{M}$
$-56.95 \mathrm{mV}$ and $-75.58 \mathrm{mV}$, respectively. Many repeated calculations indicate that our method is applicable for surface potentials within the range $\pm 100 \mathrm{mV}$. As expected the absolute value of the surface potential decreases as the ionic strength increases. We will not go into more detail since this has been amply discussed before. ${ }^{2)}$

We want now to consider the cation binding problem when a second salt is added. For this, we consider the simple model discussed in section 2 . To make it more realistic, we use the experimental data reported by Su, Chen and Mou'. They measured copper ion concentration in the presence of sodium dodecylsulfate micelles. For the system considered, $\left[M_{b}\right]_{0}=5 \times 10^{-4} M$ and $\mathrm{C}_{M}=2.4 \times 10^{-3} M$. For the three cases considered, the measured copper concentrations are (a) $\mathrm{C}_{a}=0.015 \mathrm{M}$, $\left[M_{b}\right]=2 \times 10^{-6} \mathrm{M}$; (b) $\mathrm{C}_{a}=0.01 \mathrm{M},\left[M_{b}\right]=8.17 \times$ $10^{-6} M ; \quad$ (c) $\quad \mathrm{C}_{a}=0.001 \mathrm{M}, \quad\left[M_{b}\right]=6 \times 10^{-3} \mathrm{M}$. These give (a) $\beta=2.4$, (b) $\beta=3.6$, (c) $\beta=5.4$ respectively. To proceed, we made an assumption that the aggregation number $g=70$. This number is arbitrary, but in the correct range as estimated from other similar systems ${ }^{11)}$. The value of $\alpha$ is the most uncertain. From similar studies for pure SDS, we set the $\alpha=20 \%$. We chose a higher value of $\alpha$ than in Fig. 1 because of the presence of $M_{b}$ replacing $M_{a}$. We note that this is only a model calculation, a complete predication needs a whole set of data that is not yet available in the literature. We then proceeded to calculate the surface potential as a function of $\alpha_{b}$. The results are reported in Fig. 2. As one can see, the absolute value of surface potential decreases as Stern layer binding becomes stronger $\left(\alpha_{b} \rightarrow 1\right)$. The decrease is more effective when micelle concentration is lower (curve c). At $\alpha_{b}=0$; the $\phi(a)$ are slightly different due to. the different ionic strengths considered in $\mathrm{a}, \mathrm{b}$, and $\mathrm{c}$. This indicates that the stability of the micelle against coagulation is probably lower at lower surfactant concentrations when salts are added. For some cations, 


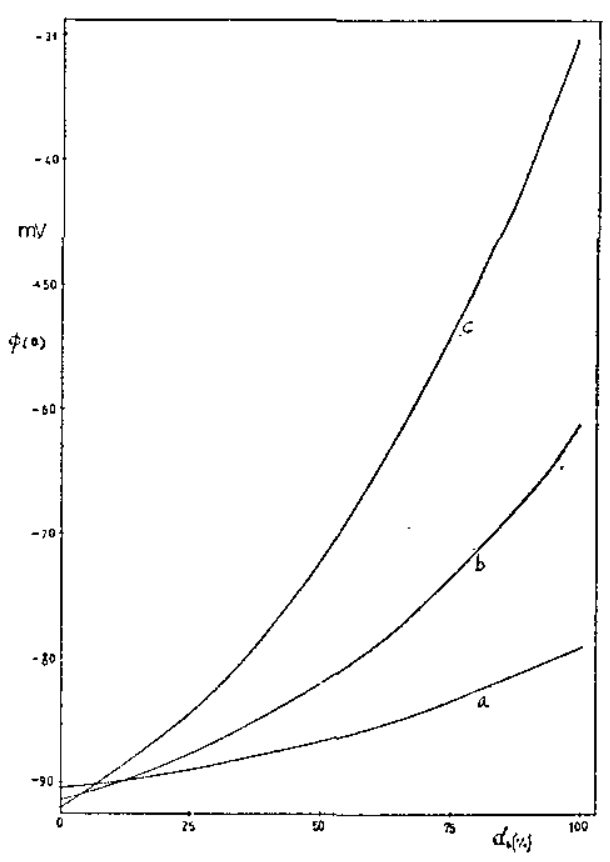

Fig. 2. Surface potential of $\phi(a)$ versus $\alpha_{b}$ at several $\beta$ values. $\left[M_{b} P\right]_{0}=5 \times 10^{-4}$ $M C_{M}=2.4 \times 10^{-3} M, \quad g=70, \quad a=$ $0.20, a=15 \AA$

Cases
(a) $\mathrm{C}_{a}=0.015 \mathrm{M},\left[\mathrm{M}_{b}\right]=2 \times 10^{-6} \mathrm{M}$
(b) $\mathrm{C}_{a}=0.01 \mathrm{M},\left[\mathrm{M}_{b}\right]=8.17 \times 10^{-6} \mathrm{M}$
(c) $\mathrm{C}_{a}=0.001 \mathrm{M},\left[\mathrm{M}_{b}\right]=6 \times 10^{-3} \mathrm{M}$

a colloid precipitation would even occur.

Up to now, we have not been able to determine the surface potential unambiguously since $\alpha_{b}$ is usually unknown. $\alpha_{b}$ cannot be determined solely from ion activities measured with electrodes because the measured effect includes both types of bindings, Stern layer and double layer. To give a complete description one needs to know the total binding of both ions on the micellar surface: This information is not easy to obtain. A promising technique to obtain this information is to measure the fluorescence quenching of a solubilized fluorephore by surface ions ${ }^{12}$.

To conclude, we have attempted an explanation of ion-micelle interaction based on a chemical exchange model on the micelle surface and a Poisson-Boltzmann equation outside the micelle. We can relate the degree of dissociation and counterion activities to the surface potential of the micelle by developing a numcerical procedure to solve the PBE. The final results indicate what kinds of experimental data are necessary for a complete understanding of the whole ionmicelle interaction behavior. We have used this method to give a partial explanation of the experimental results of Su $e t a l .^{4}$. Further work to give more quantitative explanations will be necessary in future developments.

Acknowledgement: We thank Miss Jin$\mathrm{Ru}$ Fan for preparing the manuscript. The work was supported by the National Science Council of the Republic of China.

\section{REFERENCES}

(1) J. Fendler and E. Fendler, "Catalysis in Micellar and Macromolecular Systems", Academic, New York (1975).

(2) E. Verwey and J. Th. G. Overbeek, "Theory of the Stability of Lyophobic Colloids", Elsevier, Amsterdam (1948).

(3) G. M. Bell, S. Levine and L. M. McCartney, J. Colloid Interface Sci., 33, 335 (1970).

(4) S.G. Su, Y. Chen and C.Y. Mou, Paper Submitted to J.C.C.S. (1984).

(5) A. L. Loeb, J. Th. G. Overbeek and P.H. Wiersema, "The Electrical Double Loyer Around a Spherical Particle", MIT Press, Cambridge, Mass (1961).

(6) J.G. Kirkwood, J. Chem. Phys., 2, 351 (1934).

(7) Y. Gur, J. Ravina and A. Babchin, J. Colloid Interface Sci., 64, 333 (1978).

(8) S. Levine and G.M. Bell, In "Chemical Physics of Ionic Solutions", Ed. by B.E. Conway and R.G. Barradas, Wiley, New York (1964).

(9) J. Bentz, J. Colloid Interface Sci., 80, 179 (1981).

(i0) J. Frahm and S. Diekmann, J. Colloid Interface Sci., 73, 440 (1979).

(11) L. Shedlorsky, C.W. Jakob and M. B. Epstein, J. Phys. Chem., 67, 2075 (1963).

(12) M. Almgren, P. Linse, M. Van der Auweraer, F. C. De Schryver, E. Gelade and Y. Croonen, J. Phys. Chem., 88, 289 (1984).

(Received August 22, 1984) 